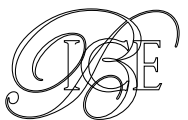

Subdirección General de Asuntos Económicos y Financieros*

\section{MARCO FINANCIERO PLURIANUAL 2021-2027 Y PLAN DE RECUPERACIÓN PARA EUROPA POS-COVID-19}

La Comisión Europea adoptó, en mayo de 2018, su propuesta para el Marco Financiero Plurianual (MFP) 2021-2027, que fue analizada, discutida y negociada en el Consejo durante las presidencias búlgara, austriaca, rumana y finlandesa sin llegarse a un acuerdo. En enero de 2020, bajo presidencia croata, el presidente del Consejo Europeo tomó las riendas de la negociación, presentando su primera propuesta de Caja de Negociación (Negotiating Box) el 14 de febrero, que fue discutida por los líderes, sin éxito, en el Consejo Europeo del 26 de marzo.

Posteriormente, las circunstancias sobrevenidas de confinamiento como consecuencia de la pandemia mundial de la COVID-19 ralentizaron las negociaciones. El impacto de la crisis en las economías de los Estados miembros llevó al Eurogrupo a acordar, el 9 de abril, una respuesta coordinada de la UE, con un paquete de medidas económicas que incluye una triple red de seguridad para las empresas, los trabajadores y los Estados por importe de 540.000 millones de euros. Por su parte, la Comisión Europea adoptó el 27 de mayo, junto con un Plan de Recuperación para Europa, que incluye un nuevo instrumento de Nueva Generación UE (Next Generation EU o NGEU), una propuesta revisada del MFP 2021-2027 ajustada a la nueva situación y una solución temporal puente para el ejercicio 2020, que implicaba modificar el MFP 2014-2020.

El 10 de julio, el presidente del Consejo Europeo, sobre la base de la propuesta de la Comisión, presentó un borrador de conclusiones integrado por su propuesta para el NGEU, el MFP 2014-2020 y la Caja de Negociación del MFP 2021-2027 ajustada, que incluía un apartado sobre los ingresos, como un paquete único e indivisible. En la madrugada del 21 de julio, el Consejo Europeo logró, tras casi cuatro días de dura negociación, un acuerdo político sobre todo el paquete.

Palabras clave: Presupuesto UE, recursos propios, instrumento de nueva generación, Consejo Europeo, Caja de Negociación.

Clasificación JEL: H30, H61, H68, H50, H72.

${ }^{*}$ Secretaria de Estado para la Unión Europea. Ministerio de Asuntos Exteriores, de la UE y Cooperación.

Este artículo ha sido elaborado por Almudena Martín-González Sánchez, Subdirectora General Adjunta; Victoria Sánchez Cifuentes, Vocal Asesor y M. ${ }^{\text {a }}$ Victoria Carcelén Ordóñez, Subdirectora General de Asuntos Económicos y Financieros. Agradecimiento especial a Antonio Castrillón.

Versión de agosto de 2020

DOI: https:/doi.org/10.32796/bice.2020.3127.7086

\section{Introducción}

El Marco Financiero Plurianual (MFP) establece los límites máximos de gasto de la UE dentro de una programación plurianual de al $\triangleright$ 
menos cinco años y garantiza la evolución ordenada de sus gastos dentro del límite de sus recursos propios.

El artículo 25 del Reglamento del Consejo 1311/2013, de 2 de diciembre de 2013, por el que se establece el MFP 2014-2020, dispone que:

Antes del 1 de enero de 2018 la Comisión presentará una propuesta de nuevo marco financiero plurianual. Si no se ha adoptado ningún reglamento del Consejo por el que se establezca un nuevo marco financiero plurianual antes del 31 de diciembre de 2020, los límites máximos y las demás disposiciones correspondientes al último año del marco financiero plurianual deberán prorrogarse hasta que se haya adoptado un reglamento que establezca un nuevo marco financiero plurianual.

Por su parte, y conforme al artículo 312.2 del Tratado de Funcionamiento de la UE:

El Consejo adoptará con arreglo a un procedimiento legislativo especial un reglamento que fije el marco financiero plurianual. El Consejo se pronunciará por unanimidad, previa aprobación del Parlamento Europeo, que se pronunciará por mayoría de los miembros que lo componen.

El próximo MFP será el primer presupuesto para una UE-27 (sin Reino Unido). Además, concurre una serie de circunstancias excepcionales a las que Europa deberá hacer frente, como los efectos de la crisis de la COVID-19, así como otros nuevos desafíos, como la crisis migratoria, el desempleo, los cambios demográfico, tecnológico o climático y la inestabilidad geopolítica.

\section{Desarrollo de las negociaciones}

Las negociaciones del MFP 2021-2027 se iniciaron formalmente el 2 de mayo de 2018, con la adopción por la Comisión Europea de su propuesta legislativa, integrada tanto por textos legislativos horizontales ${ }^{1}$ como por $\triangleright$

1 Reglamento sobre el MFP, Acuerdo Interinstitucional y Decisión del Consejo de recursos propios.

TABLA 1

ESTRUCTURA DEL MARCO FINANCIERO PLURIANUAL

\begin{tabular}{|c|c|}
\hline Rúbricas & Importe (mill. de euros) \\
\hline 1. Mercado único, innovación y digital & 166.303 \\
\hline 2. Cohesión y valores ................................ & 391.974 \\
\hline 3. Recursos naturales y medio ambiente ............... & 336.623 \\
\hline 4. Migración y gestión de fronteras ... & 30.829 \\
\hline 5. Seguridad y defensa & 24.323 \\
\hline 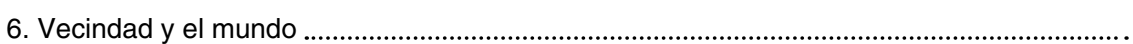 & 108.929 \\
\hline 7. Administración .............................................. & 75.602 \\
\hline 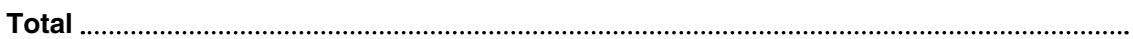 & 1.134 .583 \\
\hline
\end{tabular}


reglamentos sectoriales correspondientes a diferentes programas de gasto ${ }^{2}$.

La Comisión propuso un tamaño para el MFP por importe de 1.134 .583 millones de euros (en precios $2018^{3}$ ) en créditos de compromiso, lo que representaba el $1,114 \%$ de la renta nacional bruta (RNB) de la EU-27, con una novedosa estructura en siete rúbricas, frente a las cinco existentes en el MFP 2014-2020 (Tabla 1).

Tras una primera fase de aclaraciones técnicas por parte de la Comisión y el debate de orientación del CAG (Consejo de Asuntos Generales) en septiembre de 2018, la presidencia austriaca comenzó a trabajar a nivel técnico para identificar los elementos de la futura Caja de Negociación (Negotiating Box, NB). En noviembre de 2018 presentó una primera propuesta.

En paralelo, el Parlamento Europeo votó su posición sobre el MFP 2021-2027 el 14 de noviembre.

Durante las negociaciones los contribuyentes netos ${ }^{4}$ defendieron un tamaño del $1 \%$ de la RNB (1.018.476 millones de euros), lo que supondría reducir aproximadamente en 115.543 millones de euros la propuesta de la Comisión. Los países «amigos de un presupuesto ambicioso» 5 apoyaron el tamaño propuesto por la Comisión. El Parlamento Europeo, por su parte, defendía un tamaño de 1.324.019 millones, equivalente al $1,3 \%$ de la RNB.

En el Consejo Europeo de 13 y 14 de diciembre de 2018, los líderes tuvieron un primer debate político sobre las propuestas de la

2 Adoptados entre el 28 de mayo y el 14 de junio de 2018.

Salvo indicación en contrario, todos los importes de este artículo se refieren a precios 2018, tal y como se han negociado y recogido en la NB. Si bien las propuestas de la Comisión también se expresan referenciadas en precios corrientes.

4 Denominados cuatro frugales (Países Bajos, Suecia, Dinamarca y Austria) más Finlandia.

5 Integrado por los países cohesión: República Checa, Eslovaquia, Hungría, Lituania, Letonia, Estonia, Rumanía, Portugal, Polonia, Grecia, Bulgaria, Malta, Croacia y España.
Comisión, que esperaba mayor ambición y un impulso firme para avanzar rápidamente, con idea de fijar octubre de 2019 como fecha límite para el acuerdo. Sin embargo, las conclusiones del Consejo instaban a la presidencia entrante (rumana) a continuar con los trabajos con vistas a alcanzar un acuerdo en el Consejo Europeo en otoño de 2019.

En febrero de 2019, la presidencia rumana presentó una propuesta de simplificación de la Caja de Negociación que no tuvo buena acogida y que, tras unos meses de debates técnicos, fue presentada en el CAG de 18 de junio, siendo muy criticada por algunos Estados miembros.

Bajo presidencia finlandesa, en julio de 2019 , se entró en una nueva fase de las negociaciones. El objetivo era que en la Cumbre de los Jefes de Estado y de Gobierno de octubre hubiese un debate de orientación en profundidad sobre la base de un documento de criterios generales. El 4 de diciembre, la presidencia finlandesa presentó su propuesta de Caja de Negociación, por primera vez con cifras por políticas y programas, que fue muy criticada por la mayoría de los Estados miembros (EE MM), incluido España. Con un tamaño del $1,07 \%$ de la RNB, inferior al propuesto por la Comisión, pero muy por encima del $1 \%$ solicitado por varios EE MM, la Caja de Negociación revisada, lejos de ofrecer una solución de compromiso, alteraba el equilibrio recogido en la propuesta de la Comisión, introduciendo cambios significativos. Por citar los más importantes: el recorte de la dotación de la política de cohesión en 7.443 millones de euros, un 2,25\% menos respecto a la propuesta de la Comisión, y el hecho de que, a pesar de que se ha reforzado la PAC (Política Agraria Común), el aumento era solo en el Pilar II (desarrollo rural). 
Por otro lado, la reciente constituida nueva Comisión Europea, liderada por Ursula von der Leyen, presentó, el 11 de diciembre de 2019, su propuesta del Pacto Verde Europeo en línea con los compromisos europeos con el Acuerdo de París sobre el Clima de 2015 y con los Objetivos de Desarrollo Sostenible 2030 de la ONU.

El Consejo Europeo de 12 y 13 de diciembre, tras debatir las principales características de la Caja de Negociación presentadas por la presidencia finlandesa, invitaba al presidente del Consejo a tomar las riendas de las negociaciones con el objetivo de avanzar en las mismas.

El 14 de enero de 2020, la Comisión publicó su propuesta de Plan de Inversión del Pacto Verde Europeo, que preveía movilizar al menos un billón de euros en inversiones sostenibles en la próxima década. El plan incluía un Mecanismo de Transición Justa, que tiene como uno de sus pilares de financiación el Fondo de Transición Justa (FTJ), que recibiría 7.500 millones de euros de nueva financiación de la UE (Ilamado fresh money o dinero fresco).
El 14 de febrero, el presidente del Consejo Europeo, Charles Michel, presentó una nueva Caja de Negociación que mantenía un tamaño del 1,074 \%, incrementaba ligeramente la dotación de la rúbrica 2 y aumentaba en 7.500 millones de euros la rúbrica 3, correspondientes en su totalidad al Fondo de Transición Justa. En el Consejo Europeo extraordinario del 20 de febrero los líderes constataron que, si bien la propuesta del presidente había aproximado posiciones, existían aún elementos que dificultaban llegar al acuerdo en los términos planteados.

En marzo, las circunstancias sobrevenidas de la crisis de la COVID-19 ralentizaron las negociaciones. Los miembros del Consejo Europeo dejaron de tener reuniones presenciales y mantuvieron reuniones por videoconferencia. En concreto, en la del 23 de abril, convinieron trabajar en la creación de un Fondo de Recuperación que debía ser de una magnitud suficiente, ir dirigido a los sectores y zonas geográficas más afectados y ser específico para abordar esta crisis sin precedentes. Para ello, encomendaron a la Comisión un análisis de las necesidades exactas y la presentación $\triangleright$

TABLA 2

RÚBRICAS DEL MARCO FINANCIERO PLURIANUAL

\begin{tabular}{|c|c|c|c|c|}
\hline \multirow{2}{*}{ Rúbricas } & MFP & MFP & \multirow{2}{*}{ Diferencia } & \multirow{2}{*}{$\%$ diferencia } \\
\hline & Mayo 2018 & Mayo 2020 & & \\
\hline 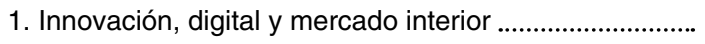 & 166.303 & 140.656 & -25.647 & $-15,42$ \\
\hline 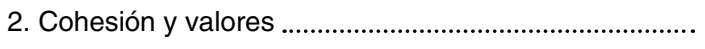 & 391.974 & 374.460 & -17.514 & $-4,47$ \\
\hline 3. Recursos naturales y medio ambiente ............................... & 336.623 & 357.032 & 20.409 & 6,06 \\
\hline 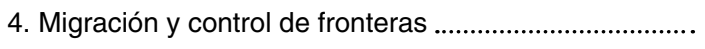 & 30.829 & 31.122 & 293 & 0,95 \\
\hline 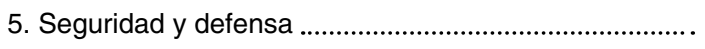 & 24.323 & 19.423 & -4.900 & $-20,15$ \\
\hline 6. Vecindad y el mundo .......................... & 108.929 & 102.705 & -6.224 & $-5,71$ \\
\hline 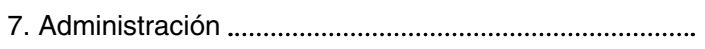 & 75.602 & 74.602 & -1.000 & $-1,32$ \\
\hline TOTAL .. & 1.134.583 & 1.100 .000 & 34.583 & $-3,05$ \\
\hline
\end{tabular}


urgente de una propuesta. Con carácter previo, España había presentado 6 , el 19 de abril, un documento con su propuesta de creación de un fondo de recuperación económica para atender los gastos derivados de los efectos de la crisis de la COVID-19 por un importe de 1.500.000 millones de euros, financiado con deuda y que otorgase transferencias a los Estados miembros.

El 27 de mayo, la Comisión propuso su Plan de Recuperación de la UE por importe de 1.850 .000 millones de euros con el fin de movilizar las inversiones necesarias. El plan supone una respuesta doble a la crisis a través de:

- El instrumento Next Generation EU (NGEU, Unión Europea de Nueva Generación), cuyo objetivo es ayudar a la recuperación económica a través de inversiones y reformas, así como impulsar el presupuesto de la UE con nueva financiación obtenida de los mercados financieros por un importe de 750.000 millones de euros, que se instrumenta a través de una serie de programas de nueva creación o ya existentes en la propuesta inicial de la Comisión.

- El presupuesto a largo plazo de la Unión Europea reforzado para el periodo 20212027. El MFP se incrementa hasta 1.100 .000 millones de euros, algo más ambicioso que en las últimas negociaciones.

El Plan de recuperación de la Comisión incluye también un refuerzo en el MFP 20142020 para el año 2020 por importe de 11.500 millones de euros, como una solución

6 Otros Estados miembros, como Francia, Alemania o Países Bajos, también circularon propuestas al respecto. temporal puente a la crisis de la COVID-19 y que complementa las medidas previamente propuestas por la Comisión en marzo, en su comunicación sobre la respuesta económica coordinada al brote de COVID-19. Entre ellas, se incluye la Iniciativa de Inversión dentro de la política de cohesión (Coronavirus Response Investment Iniciative -CRII-), que implica destinar 37.000 millones de euros de la política de cohesión a la lucha contra la crisis del coronavirus?.

En la videoconferencia del 19 de junio los miembros del Consejo Europeo debatieron la propuesta sin que se produjesen avances.

Sobre la base de la propuesta de la Comisión, el presidente del Consejo Europeo presentó, el 10 de julio, un borrador de conclusiones que incluía su propuesta de paquete único integrado por la propuesta del NGEU, una versión revisada de la Caja de Negociación del MFP 2021-2027, la propuesta de la Comisión para el MFP 2014-2021 y una propuesta sobre el sistema de financiación de la UE.

El 17 de julio los jefes de Estado y de Gobierno de la UE asistieron presencialmente a uno de los Consejos europeos más largos que se recuerdan. El objetivo estaba claro: era imprescindible alcanzar un acuerdo. Europa tenía que enviar una señal a sus ciudadanos, a los mercados y al mundo. Tras casi cuatro días de duras negociaciones, la madrugada del día 21 se anunciaba, por el presidente del Consejo Europeo, el acuerdo político unánime de sus miembros sobre el paquete global.

En el Gráfico 1 se recoge el reparto entre rúbricas comparado entre las diferentes propuestas de la Comisión Europea y las Cajas de negociación.

7 Posteriormente, se adoptaría el CRII+, que incluye, entre otras, medidas de flexibilización en el uso de los fondos de la política de cohesión. 


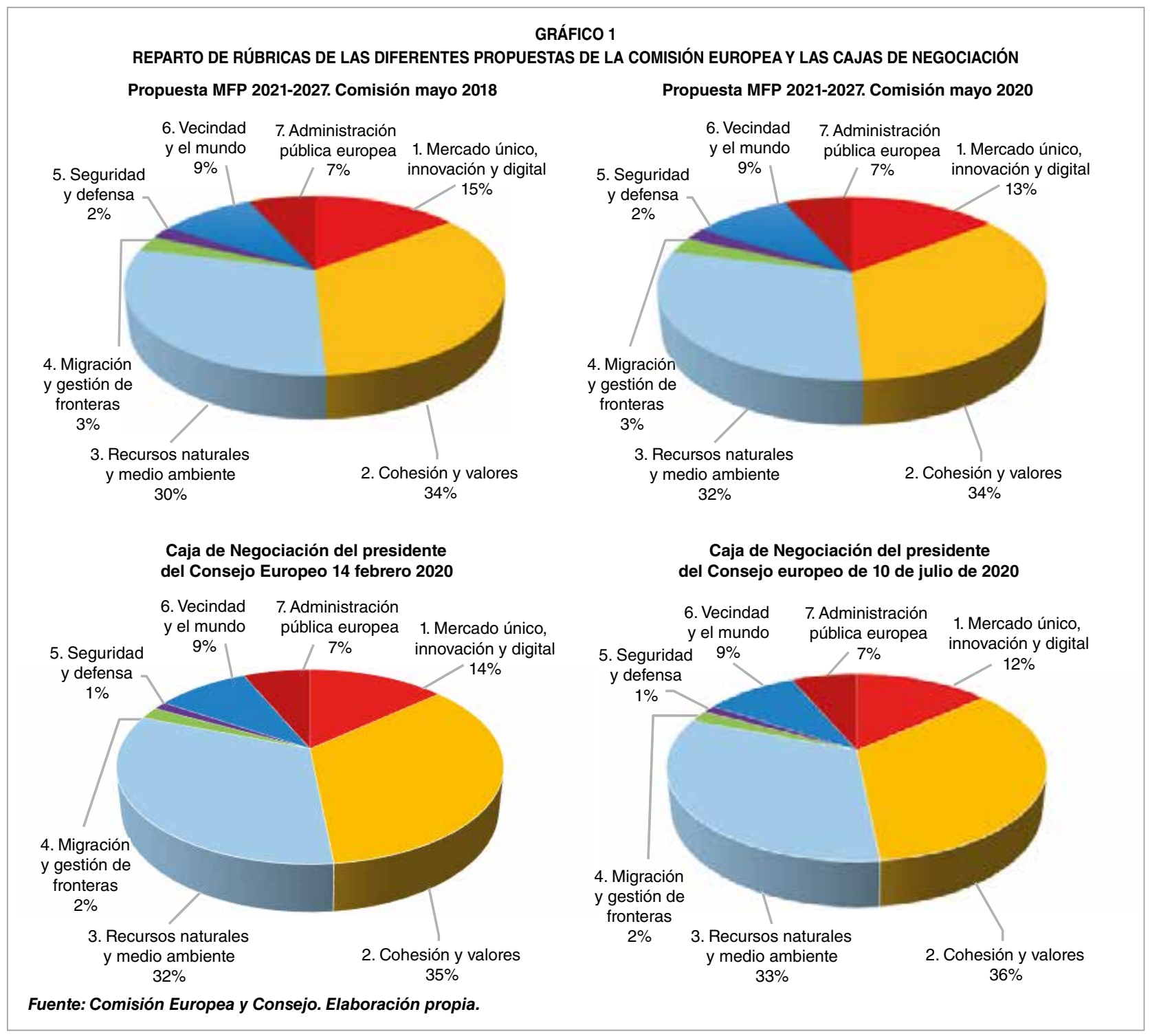

\section{Contenido de las conclusiones del Consejo Europeo de 21 de julio}

Los líderes acordaron que no habría cambios en el MFP 2014-2020, por lo tanto, la solución puente propuesta por la Comisión quedaba descartada.

\subsection{Instrumento de nueva generación EU (NGEU)}

Constituye un elemento novedoso, temporal y excepcional. Estará dotado con 750.000 millones de euros (el mismo importe propuesto por la Comisión), repartido en 360.000 para préstamos y 390.000 para subvenciones (frente a la propuesta de la Comisión de 500.000 subvenciones y 250.000 préstamos), cuya finalidad es facilitar la recuperación económica de los países o sectores más afectados por la crisis de la COVID-19. Para su financiación la Comisión estará autorizada a pedir prestados fondos en nombre de la Unión en los mercados de capitales. Los ingresos se transferirán a los siguientes programas de la Unión, dirigidos a: 
Apoyar a los Estados miembros

- Facilidad de recuperación y resiliencia, con una dotación de 672.500 millones de euros que se utilizará para inversiones y reformas en relación con las transiciones ecológicas y digitales y la resiliencia de las economías nacionales para una recuperación y resiliencia duraderas, en consonancia con los objetivos identificados en el Semestre Europeo.

- ReactEU, orientado a aumentar la dotación de cohesión, con las condiciones flexibles establecidas para las iniciativas CRII y CRII+. Estos fondos se destinarán a subvenciones a municipios, hospitales, empleo, planes de trabajo a corto plazo y a liquidez y solvencia de las pymes. La iniciativa React-UE proporcionará 47.500 millones de euros de financiación adicional de la política de cohesión.

- Desarrollo rural, con un refuerzo de 7.500 millones y el Fondo de Transición Justa con un importe de 10.000 millones de euros.
Poner en marcha la economía de la UE mediante el incentivo de las inversiones privadas

- Fortalecimiento de InvestEU, con un incremento de hasta 5.600 millones para las cuatro ventanas operativas ya acordadas por los colegisladores.

Aprender las lecciones de la crisis y abordar los desafíos estratégicos de Europa

- Un aumento de hasta 1.500 millones para RescEU, el mecanismo de protección civil de la UE, que se ampliará y reforzará para dotar a la Unión de una mejor capacidad para responder a futuras crisis de manera flexible.

- Un incremento de hasta 5.000 millones en Horizonte Europa, con el objetivo de aumentar el apoyo europeo a las actividades de investigación e innovación relacionadas con salud, clima, resiliencia y transiciones verdes y digitales.

En la Tabla 3 se detalla la comparativa sobre la propuesta de la Comisión y el acuerdo final del NGEU.

TABLA 3

COMPARATIVA SOBRE LA PROPUESTA DE LA COMISIÓNY EL ACUERDO FINAL DEL NGEU

\begin{tabular}{|c|c|c|c|c|}
\hline $\begin{array}{l}\text { Next Generation EU } \\
\text { (Millones de euros a precios de 2018) }\end{array}$ & $\begin{array}{c}\text { Propuesta } \\
\text { Comisión } 27 / 05 / 20\end{array}$ & $\begin{array}{l}\text { Conclusiones adoptadas } \\
\text { por el Consejo } 21 / 07 / 20\end{array}$ & $\begin{array}{l}\text { Difer } \\
\text { impc }\end{array}$ & $\begin{array}{l}\text { ncias } \\
\text { tel } \%\end{array}$ \\
\hline Facilidad de recuperación y resiliencia transferencias ..... & 310.000 & 360.000 & 50.000 & 16,13 \\
\hline Facilidad de recuperación y resiliencia préstamos ............. & 250.000 & 312.500 & 62.500 & 25,00 \\
\hline React EU & 50.000 & 47.500 & -2.500 & $-5,00$ \\
\hline Horizon Europe & 13.500 & 5.000 & -8.500 & $-62,96$ \\
\hline Invest EU & 30.300 & 5.600 & -24.700 & $-81,52$ \\
\hline Instrumento de apoyo a la solvencia .... & 26.000 & 0 & -26.000 & $-100,00$ \\
\hline Fondo Europeo Agrícola de Desarrollo Rural ......................... & 15.000 & 7.500 & -7.500 & $-50,00$ \\
\hline Fondo de Transición Justa & 30.000 & 10.000 & -20.000 & $-66,67$ \\
\hline 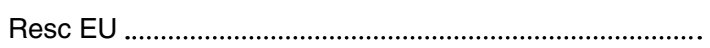 & 2.000 & 1.900 & -100 & $-5,00$ \\
\hline Programa de salud & 7.700 & 0 & -7.700 & $-100,00$ \\
\hline $\mathrm{NDCl}$ & 15.500 & 0 & -15.500 & $-100,00$ \\
\hline 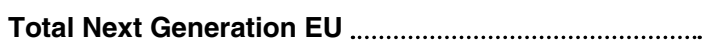 & 750.000 & 750.000 & 0 & 0,00 \\
\hline
\end{tabular}


Las mayores dificultades para encontrar consenso durante las negociaciones se centraron en el importe total del instrumento, así como en la determinación de las cantidades que se destinarían para préstamos y subvenciones. Por otro lado, en relación a la facilidad de recuperación y resiliencia, las dificultades se centraron en el procedimiento y la condicionalidad exigida en los planes de recuperación y resiliencia para otorgar la financiación a los Estados miembros. En ambos aspectos la posición defendida por los países frugales encontró firme oposición por parte de España y otros países como Italia y Francia, llegándose finalmente a una solución de compromiso. Los planes de recuperación y resiliencia serán evaluados por la Comisión bajo criterios de coherencia con las recomendaciones específicas por país, así como el fortalecimiento del potencial de crecimiento, la creación de empleo y la resiliencia económica y social, y la contribución efectiva a la transición verde y digital. La evaluación de los planes será aprobada por el Consejo, por mayoría cualificada a propuesta de la Comisión.

Para la financiación del instrumento, es necesario modificar la decisión de recursos propios e incrementar temporalmente los límites máximos de los recursos propios en 0,6 puntos porcentuales. El reembolso se programará, de conformidad con el principio de buena gestión financiera, para garantizar una reducción constante y previsible de los pasivos hasta el 31 de diciembre de 2058.

\subsection{Partida de gastos}

El importe total para el periodo 2021-2027 asciende a 1.074.300 millones de euros en créditos compromisos, incluido el Fondo Europeo de Desarrollo (que en el MFP 2014-2020 se sitúa fuera de los techos de gasto), y 1.061 .058 millones de euros en créditos para pagos. En la Tabla 4 se recoge una comparativa entre la propuesta de la Comisión de mayo de 2020 y el acuerdo político del 21 de julio.

Se trata de un presupuesto UE:

- Con una estructura más transparente, en la que los programas se agrupan en torno a prioridades temáticas de gasto $y$ existe una vinculación entre los ingresos y las políticas de gasto que financian. Más ágil y flexible, se reduce el número de instrumentos especiales y crea una nueva reserva de ayuda solidaria y de emergencia con un importe anual de $\triangleright$

TABLA 4

COMPARATIVA ENTRE LA PROPUESTA DE LA COMISIÓN Y LAS CONCLUSIONES DEL CONSEJO EUROPEO

\begin{tabular}{|c|c|c|c|c|}
\hline $\begin{array}{l}\text { Marco Financiero Plurianual. Rúbricas } \\
\text { (Millones de euros a precios de 2018) }\end{array}$ & $\begin{array}{l}\text { Propuesta Comisión } \\
27 / 05 / 2020\end{array}$ & $\begin{array}{l}\text { Conclusiones del Consejo Europeo } \\
21 / 07 / 2020\end{array}$ & \multicolumn{2}{|c|}{$\begin{array}{l}\text { Diferencia } \\
\text { importe } / \%\end{array}$} \\
\hline 1. Innovación, digital y mercado interior .... & 140.656 & 132.781 & -7.875 & $-5,60$ \\
\hline 2. Cohesión y valores & 374.460 & 377.768 & 3.308 & 0,88 \\
\hline 3. Recursos naturales y medio ambiente.. & 357.032 & 356.374 & -658 & $-0,18$ \\
\hline 4. Migración y control de fronteras ................ & 31.122 & 22.671 & -8.451 & $-27,15$ \\
\hline 5. Seguridad y defensa & 19.423 & 13.185 & -6.238 & $-32,12$ \\
\hline 6. Vecindad y el mundo & 102.705 & 98.419 & -4.286 & $-4,17$ \\
\hline 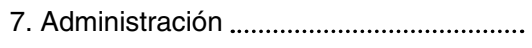 & 74.602 & 73.102 & -1.500 & $-2,01$ \\
\hline TOTAL .. & 1.100 .000 & 1.074 .300 & -25.700 & $-2,34$ \\
\hline
\end{tabular}

Fuente: Comision Europea y Consejo. 
1.200 millones de euros (fusionando dos instrumentos existentes en el MFP anterior). Además, crea una reserva de 5.000 millones de euros para atender las consecuencias del Brexit en los sectores y Estados más afectados. El importe total de los instrumentos cuyos créditos se sitúan fuera de los techos del MFP ascenderá a 20.106 millones de euros. No habrá revisión a medio plazo, inicialmente prevista en la propuesta de la Comisión.

- Con medidas adicionales de flexibilidad, relativas a las normas de ejecución en el marco de la política de cohesión y de la PAC, así como a la concentración temática de la ayuda del Fondo Europeo de Desarrollo Regional (FEDER).

- Basado en el rendimiento y el cumplimiento con nuevas condicionalidades, como la climática (se fija el objetivo del $30 \%$ del total de gasto en clima), y refuerzo de las ya existentes, como la económica o el cumplimiento del Estado de derecho, que se extiende también al mecanismo NGEU.

- Reparto entre rúbricas: el mayor volumen de financiación se concentra en las rúbricas 2 , Cohesión, resiliencia y valores, y 3, Recursos naturales y medioambiente, que representan cerca del $70 \%$ del total del MFP.

- La rúbrica 1, Mercado único, innovación y digital, tiene una dotación de 132.781 millones, manteniendo las asignaciones propuestas de febrero 2020 para el Programa Espacial en 13.202 millones, de los cuales 8.000 son para financiar el programa Galileo y 4.810 para financiar el programa Copérnico. Al programa Horizonte Europa se destinarán 75.900 .
- En la rúbrica 2 el importe global alcanza los 377.768 millones de euros. Se crean dos subrúbricas, de tal manera que 330.235 millones serán para la subrúbrica de cohesión y 47.533 millones para la subrúbrica de resiliencia y valores.

Los recursos para el objetivo «Inversión para el empleo y crecimiento» ascienden hasta los 322.285 millones, de los cuales 202.299 serán para las regiones menos desarrolladas, 47.789 para las regiones en transición, 27.212 para las regiones más desarrolladas, 42.556 para Estados miembros apoyados por el Fondo de Cohesión, 1.928 de fondos adicionales para las regiones ultraperiféricas (RUP) - de los que se beneficiará la Comunidad Autónoma de Canarias- y 500 para inversión en innovación interregional.

La dotación del Fondo Social Europeo alcanza los 87.319 millones de euros, incluyendo financiación específica por valor de 473 millones para las RUP y las regiones poco pobladas del norte; 175 millones de los recursos del Fondo Social Europeo (FSE) bajo el objetivo "Inversión para el empleo y crecimiento" se destinarán a la cooperación trasnacional para apoyar soluciones innovadoras bajo gestión directa o indirecta. En lo que respecta a la resiliencia, se dota al programa RescUE con 1.106 millones y el programa de Salud con 1.670 millones de euros.

- La rúbrica 3, Recursos naturales y medio ambiente, tiene una dotación de 356.374 millones destinados a los objetivos de la política agrícola común (PAC) y la política pesquera, así $\square$ 
como del Programa de Acción por el Clima y el Medio Ambiente. De este importe, 258.594 millones se asignarán al Pilar I (gastos de mercado y pagos directos). Por otra parte, el Fondo de Transición Justa se cifra en 7.500 millones. Además, para aquellos Estados miembros ${ }^{8}$ que se enfrentan a desafíos estructurales particulares en su sector agrícola, hayan invertido fuertemente en el gasto del Pilar II o necesiten transferir importes más altos al Pilar I para aumentar el grado de convergencia, dentro del importe global, se asignan dotaciones adicionales.

- El resto de rúbricas representan un volumen total inferior al $20 \%$ del total del MFP. Se crean dos nuevas rúbricas, que reflejan las prioridades políticas de gasto de la UE. La rúbrica 4, Migración y control de fronteras, está dotada con 22.671millones de euros, y la rúbrica 5 , Seguridad y Defensa, con 13.185 millones, de los cuales 7.014 millones corresponden al Fondo Europeo de Defensa, 1.705 al Fondo de Seguridad Interna y 1.500 al Mecanismo conectar Europa (CEF por sus siglas en inglés) para el movimiento de tropas. La rúbrica 6 , Vecindad y el mundo, dotada con 98.419 millones (frente a 102.705 de la propuesta de la Comisión), incluye el novedoso Instrumento de vecindad, desarrollo y cooperación internacional (NDICl, por sus siglas en inglés), con una dotación de 70.800 millones, de los cuales el $76 \%$ estará destinado a programas geográficos.

8 Bélgica, Alemania, Irlanda, Grecia, Francia, Croacia, Italia, Chipre, Malta, Austria, Eslovaquia, Eslovenia, Portugal, Finlandia y España (con una asignación de 500 millones de euros).

\subsection{Partida de ingresos}

Se propone que el $25 \%$ de los costes de recaudación de los recursos propios tradicionales sea para los EE MM (la propuesta de la Comisión era del $10 \%$, el régimen vigente es del $20 \%$ y en la propuesta de febrero el $12,5 \%$ ). El recurso propio actual basado en el IVA será sustituido por el método simplificado y mejorado de la Comisión presentado en enero de 2019 , con un tipo uniforme del $0,3 \%$.

En relación al establecimiento de nuevos recursos propios, en una primera etapa se instaurará uno nuevo basado en los residuos plásticos no reciclados, que se aplicará a partir del 1 de enero de 2021. En el primer semestre de 2021, la Comisión presentará propuestas relativas a un mecanismo de ajuste en frontera de las emisiones de carbono y a un impuesto digital, que servirán de base para recursos propios adicionales con vistas a su instauración a más tardar el 1 de enero de 2023. En la misma línea, la Comisión presentará una propuesta de revisión del régimen de comercio de derechos de emisión que podría hacerse extensiva al transporte aéreo y marítimo. Por último, en el transcurso del próximo MFP la Unión trabajará para instaurar otros recursos propios, entre los que podría incluirse un impuesto sobre las transacciones financieras. Los ingresos generados por los nuevos recursos propios introducidos después de 2021 se utilizarán para el reembolso anticipado de los préstamos del NGEU.

En cuanto a los mecanismos de corrección, para el periodo 2021-2027 están previstas correcciones a tanto alzado que reducirán la contribución anual basada en la RNB de Dinamarca, Alemania, Países Bajos, Austria y Suecia. A este respecto cabe indicar que la propuesta inicial de la Comisión incluía un régimen $\triangleright$ 
transitorio de compensaciones durante los primeros cinco años para su posterior supresión, teniendo en cuenta que la salida de Reino Unido de la UE y la consiguiente desaparición del cheque británico constituía una oportunidad para que las correcciones desaparecieran.

\section{Valoración para España}

La valoración del resultado del Consejo europeo del 21 de julio, tanto en relación con el MFP 2021-2027 como sobre el instrumento NGEU, es positiva para España. Con respecto a este último, se ha mantenido el importe inicialmente propuesto por la Comisión de 750.000 millones de euros, así como un importe significativo de transferencias, superior al de los préstamos, frente a las peticiones de los frugales de su reducción. España será uno de los países más beneficiados con la financiación del NGEU. Recibirá del instrumento ayudas por importe de 140.000 millones de euros, 72.700 millones en ayudas directas y 67.300 millones en forma de préstamos. Las subvenciones que se otorgarán a través de la Facilidad de resiliencia y recuperación ascenderán a 59.000 millones, y a través del ReactEu ascenderán a 12.400 millones. Por lo que respecta al MFP 2021-2027, el conjunto de las intervenciones de la Política Agrícola Común en forma de ayudas directas, de desarrollo rural y de mercado van a tener la misma cuantía que en el marco financiero plurianual 2014-2020.

\section{Próximos pasos}

Una vez fijada la posición del Consejo, deben retomarse las negociaciones con el
Parlamento Europeo con objeto de finalizar los trabajos relativos a todos los textos legislativos, de conformidad con las bases jurídicas pertinentes y con carácter excepcionalmente urgente para garantizar que la UE pueda responder a la crisis con rapidez y la financiación esté disponible a tiempo.

Por otro lado, será necesario que en cuanto se adopte la decisión sobre los recursos propios, los Estados miembros procedan a su aprobación lo antes posible, de conformidad con sus respectivos requisitos constitucionales, a efectos de su entrada en vigor, que tendrá efectos retroactivos.

El objetivo de la presidencia alemana del Consejo es alcanzar un acuerdo entre las tres instituciones sobre el paquete global antes de final de año, a efectos de garantizar una transición adecuada entre marcos y una efectiva puesta a disposición de los EE MM, en 2021, de la financiación necesaria para su recuperación.

\section{Bibliografía}

Comisión Europea (2012). Versión consolidada del Tratado de Funcionamiento de la Unión Europea. 2012 C 326/47. Bruselas: Diario Oficial de la Unión Europea. http://eur-lex.europa.eu/legalcontent/ES/TXT/PDF/?uri=CELEX:12012E/TXT \&from $=E S$

Comisión Europea (2018). Paquete legislativo de 2 de mayo de 2018, integrado por los documentos COM 321, 322, 323, 324, 325, 326, 327 +ADD. https://ec.europa.eu/commission/future-europe/eu-budget-future_en

Comisión Europea (2020). Paquete legislativo de 24 de mayo de 2020, integrado por los documentos COM 442, 443, 44, 445, 446 + ADD. https:// ec.europa.eu/info/publications/mff-legislation en 
Comisión Europea (2020) COM 456. Comunicación: El momento de Europa: reparar los daños y preparar el futuro para la próxima generación. https://eur-lex.europa.eu/legal-content/ES/TXT/ PDF/?uri=CELEX:52020DC0456\&from=EN

Comisión Europea (2019). El Pacto Verde Europeo. Bruselas. https://ec.europa.eu/commission/ presscorner/detail/es/ip_19_6691

Comisión Europea (2020). Financiar la transición verde: el Plan de Inversiones del Pacto Verde Europeo y el Mecanismo para una Transición Justa. Bruselas. https://ec.europa.eu/regional_ policy/es/newsroom/news/2020/01/14-01-2020financing-the-green-transition-the-europeangreen-deal-investment-plan-and-just-transitionmechanism

Comisión Europea (2020). Cohesion Policy and EU Solidarity Fund contribute to the Coronavirus Response Investment Initiative. Bruselas. https://ec.europa.eu/regional_policy/en/newsroom/news/2020/03/16-03-2020-cohesion-policyand-eu-solidarity-fund-contribute-to-the-coronavirus-response-investment-initiative

Consejo (2020) COM 441. Reglamento del Consejo por el que se establece un Instrumento de Recuperación de la Unión Europea para apoyar la recuperación tras la pandemia de COVID-19. https://eur-lex.europa.eu/legal-content/ES/TXT/ PDF/?uri=CELEX:52020PC0441R(01)\&from $=E N$

Consejo Europeo (21 de julio de 2020). Conclusiones del Consejo europeo. https://www.consilium.europa.eu/media/45124/210720-euco-final-conclusions-es.pdf

\section{Páginas web consultadas:}

Comisión Europea (2017). The Budget explained. Bruselas. http://ec.europa.eu/budget/explained/ glossary/glossary_en.cfm

Consejo Europeo. https://www.consilium.europa.eu/ es/policies/the-eu-budget/long-term-eu-budget2021-2027/

Consejo Europeo (23 de abril de 2020). Videoconferencia de los miembros del Consejo Europeo. https://www.consilium.europa.eu/es/meetings/ european-council/2020/04/23/

Consejo Europeo (19 de junio de 2020). Videoconferencia de los miembros del Consejo Europeo. https://www.consilium.europa.eu/es/meetings/ european-council/2020/06/19/

Darvas, Z. (29 de mayo de 2020). An uncompromising Budget. Bruegel. https://european.economicblogs.org/bruegel/2020/darvas-uncompromisingbudget

Feas, E. (2020). Las cifras del Plan de Recuperación para Europa. Real Instituto Elcano. http://www.realinstitutoelcano.org/wps/portal/rielcano_es/contenido?WCM_GLOBAL_CONTEXT=/elcano/elcano_es/zonas_es/ari86-2020-feas-cifras-del-plan-derecuperacion-para-europa

Kölling, M. (2020). Solidaridad y condicionalidad europea en tiempos pos-COVID-19. Real Instituto Elcano. http://www.realinstitutoelcano.org/wps/ portal/rielcano_es/contenido?WCM_GLOBAL_ CONTEXT=/elcano/elcano_es/zonas_es/ ari78-2020-kolling-solidaridad-y-condicionalidad-europea-en-tiempos-post-COVID-19 


\section{Anexos}

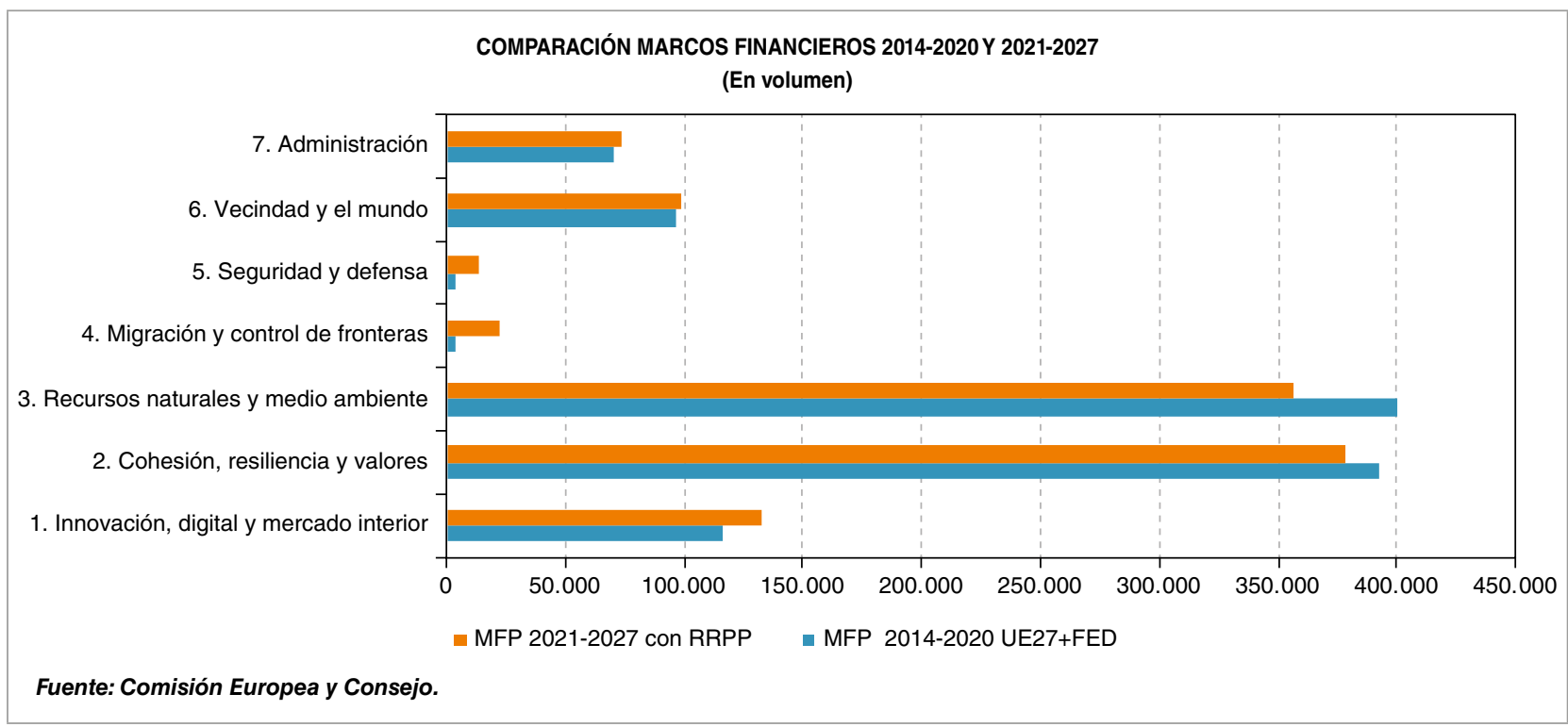

MARCO FINANCIERO PLURIANUAL 2021-2027 (EU27) (Millones de euros. Precios 2018)

\begin{tabular}{|c|c|c|c|c|c|c|c|c|}
\hline Créditos de compromiso & 2021 & 2022 & 2023 & 2024 & 2025 & 2026 & 2027 & Total \\
\hline 1. Mercado único, innovación y digital ...................... & 19.712 & 19.666 & 19.133 & 18.633 & 18.518 & 18.646 & 18.473 & 132.781 \\
\hline 2. Cohesión, resiliencia y valores ............................... & 49.741 & 51.101 & 52.194 & 53.954 & 55.182 & 56.787 & 58.809 & 377.768 \\
\hline 2a. Cohesión social, económica y territorial .. & 45.411 & 45.951 & 46.493 & 47.130 & 47.770 & 48.414 & 49.066 & 330.235 \\
\hline 2b. Resiliencia y valores ....... & 4.330 & 5.150 & 5.701 & 6.824 & 7.412 & 8.373 & 9.743 & 47.533 \\
\hline 3. Recursos naturales y medio ambiente ................. & 55.242 & 52.214 & 51.489 & 50.617 & 49.719 & 48.932 & 48.161 & 356.374 \\
\hline $\begin{array}{l}\text { de los cuales: gasto relacionado con los } \\
\text { mercados y pagos directos }\end{array}$ & 38.564 & 38.115 & 37.604 & 36.983 & 36.373 & 35.772 & 35.183 & 258.594 \\
\hline 4. Migración y gestión de fronteras ... & 2.324 & 2.811 & 3.164 & 3.282 & 3.672 & 3.682 & 3.736 & 22.671 \\
\hline 5. Seguridad y defensa ........ & 1.700 & 1.725 & 1.737 & 1.754 & 1.928 & 2.078 & 2.263 & 13.185 \\
\hline 6. Vecindad y el mundo ...... & 15.309 & 15.522 & 14.789 & 14.056 & 13.323 & 12.592 & 12.828 & 98.419 \\
\hline 7. Administración publica europea ....... & 10.021 & 10.215 & 10.342 & 10.454 & 10.554 & 10.673 & 10.843 & 73.102 \\
\hline $\begin{array}{l}\text { de los cuales: gasto administrativo de las } \\
\text { Instituciones }\end{array}$ & 7.742 & 7.878 & 7.945 & 7.997 & 8.025 & 8.077 & 8.188 & 55.852 \\
\hline Total créditos de compromiso & 154.049 & 153.254 & 152.848 & 152.750 & 152.896 & 153.390 & 155.113 & 1.074 .300 \\
\hline Total créditos de pago & 156.557 & 154.822 & 149.936 & 149.936 & 149.936 & 149.936 & 149.936 & 1.061 .058 \\
\hline
\end{tabular}

